\title{
Two-Dimensional Frank-Kasper $Z$ Phase with One Unit-Cell Thickness
}

\section{Supplementary Information}

Hongbo Xie ${ }^{\dagger}$, Junyuan Bai ${ }^{\dagger}$, Haiyan Ren ${ }^{\dagger}$, Shanshan $\mathrm{Li}^{\dagger}$, Hucheng $\operatorname{Pan}^{\dagger} *$, Yuping Ren ${ }^{\ddagger} \S *$, Gaowu Qin ${ }^{\ddagger}$

${ }^{\dagger}$ Key Laboratory for Anisotropy and Texture of Materials (Ministry of Education), School of Materials Science and Engineering, Northeastern University, Shenyang 110819, China

$\ddagger$ State Key Laboratory of Rolling and Automation, Northeastern University, Shenyang 110819, China $\S$ Research Center for Metal Wires, Northeastern University, Shenyang 110819, China

*Corresponding authors. E-mail: panhc@atm.neu.edu.cn; renyp@atm.neu.edu.cn; qingw@smm.neu.edu.cn. 


\section{Materials and Methods}

\subsection{Experimental procedure}

The alloy with a nominal composition of $\mathrm{Mg}-4.0 \mathrm{wt} \% \mathrm{Sm}-2.0 \mathrm{wt} \% \mathrm{Zn} \quad(\mathrm{Mg}-0.68 \mathrm{at} . \% \mathrm{Sm}-$ 0.78at.\%Zn) was prepared by melting the pure $\mathrm{Mg}(99.9 \mathrm{wt} \%)$, pure $\mathrm{Zn}(99.9 \mathrm{wt} \%)$, and $\mathrm{Mg}-25 \mathrm{Sm}$ (wt\%) master alloy in the induction furnace with protection of the argon atmosphere. The molten alloy was stirred and kept at $760{ }^{\circ} \mathrm{C}$ for 5 minutes and poured into a steel mold preheated to $300{ }^{\circ} \mathrm{C}$. The chemical composition of as-cast ingot was measured by OPTIMA 4300 DV composition analyzer, and the actual composition was determined to be $\mathrm{Mg}-4.12 \mathrm{wt} \% \mathrm{Sm}-2.07 \mathrm{wt} \% \mathrm{Zn}$. The as-cast samples were solution treated at $520{ }^{\circ} \mathrm{C}$ for $12 \mathrm{~h}$, followed by water quenching and ageing in oil bath at $200{ }^{\circ} \mathrm{C}$ for different times, and finally continued isothermally aged at $250{ }^{\circ} \mathrm{C}$ for $2 \mathrm{~h}$.

The small-angle X-ray scattering (SAXS) experiment was performed using synchrotron radiation as the X-ray source at the 1W2A station at the Beijing Synchrotron Radiation Facility (BSRF). The incident X-ray wavelength $\lambda$ was $0.1033 \mathrm{~nm}(12 \mathrm{keV})$. The scattering signals were collected using a Mar $165 \mathrm{CCD}$, which was positioned $1550 \mathrm{~mm}$ away from the sample. And the exposure time was 20 s. The scattering vector magnitude $q$ ranged from 0.002 to $3.133 \mathrm{~nm}^{-1}$ for the experiment reported in this paper. The two-dimensional SAXS images were transferred to one-dimensional data by using the Fit2D software of the European Synchrotron Radiation Facility.

The TEM specimens with a diameter of $3 \mathrm{~mm}$ were prepared by twin jet electro-polishing at - 40 ${ }^{\circ} \mathrm{C}$ in mixture solution of $5.3 \mathrm{~g}$ lithium chloride, $11.2 \mathrm{~g}$ magnesium perchlorate, $500 \mathrm{ml}$ methanol and $100 \mathrm{ml}$ 2-butoxy ethanol, and subsequently ion milling with low energy electron beam. Finally, Gatan SOLARUS (950) Plasma Cleaning System was used to clean up the sample surfaces. TEM and STEM observation was carried out by using the JEM-ARM200F at an accelerating voltage of $200 \mathrm{kV}$, equipped with probe Cs corrector and cold field emission gun. The probe convergence is $25 \mathrm{mrad}$ which yields a probe size of less than $0.1 \mathrm{~nm}$, and the camera length was set to $8 \mathrm{~cm}$ which yields a collection semi-angle of 48-327 mrad.

Crystal structures were reconstructed by the CrystalMaker.CrystalMaker.v2.2.4 software, and then the electron diffraction patterns of the precipitated phase detected from the [0001], [1 100$]$, and [11̄0] directions were simulated by using CrystalMaker.SingleCrystal.v2.0.1.

\subsection{Computational methods}

The first-principles calculations were conducted using the Vienna ab-initio simulation package (VASP) ${ }^{1}$ with projector augmented-wave (PAW) ${ }^{2,3}$ pseudopotentials. The exchange-correlation potential was described by the generalized gradient approximation (GGA) functional of Perdew, Burke and Ernzerhof (PBE) ${ }^{4}$. A high plane-wave cutoff energy of $500 \mathrm{eV}$ and dense $k$-point sampling based Gamma-centered Monkhorst-pack scheme are employed to guarantee high numerical accuracy. 
The geometry optimization process were performed using a conjugate gradient algorithm until the final force on each atom was less than $0.01 \mathrm{eV} / \AA$.

The formation energy, $E_{f}$, can approximately characterize the stability of compounds in thermodynamics ${ }^{5}$. And the formation energies in present calculations, $E_{f}$, were calculated as following equation:

$$
E_{f}=\frac{E\left(M g_{x} S \mathrm{~m}_{y} Z \mathrm{n}_{z}\right)-x * E\left(M g_{x}\right)-y * E\left(S \mathrm{~m}_{y}\right)-z * E(Z \mathrm{n})}{x+y+z}
$$

Here, $E\left(M g_{x} S m_{y} Z n_{z}\right), E(M g), E(S m)$ and $E(Z n)$ represent the total energy of the model, the energies of $\mathrm{Mg}, \mathrm{Sm}$ and $\mathrm{Zn}$ unit cell, respectively. The charge distribution and Electron Localized Function (ELF) models were displayed by using the VESTA software.

An open visualization tool (OVITO software package) was used for Voronoi analysis.

\section{References}

(1) Kresse, G.; Furthmüller, J. Efficient iterative schemes for ab initio total-energy calculations using a plane-wave basis set. Phys. Rev. B 1996, 54, 11169.

(2) Blöchl, P. E. Projector augmented-wave method. Phys. Rev. B 1994, 50, 17953.

(3) Kresse, G.; Joubert, D. From ultrasoft pseudopotentials to the projector augmented-wave method. Phys. Rev. B 1999, 59, 1758.

(4) Perdew, J. H.; Burke, K.; Ernzerhof, M. Generalized gradient approximation made simple. Phys. Rev. Lett. 1996, $77,3865$.

(5) Manzoor, A.; Pandey, S.; Chakraborty, D.; Phillpot, S. R.; Aidhy, D. S. Entropy contributions to phase stability in binary random solid solutions. Npj Comput. Mater. 2018, 4, 1-10. 


\section{Supplementary Figures}

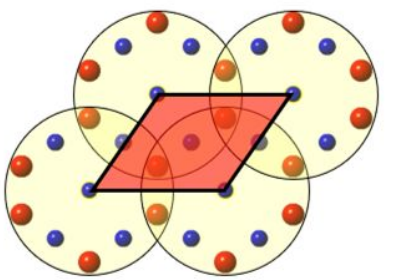

$C_{15}$-phase, [110]

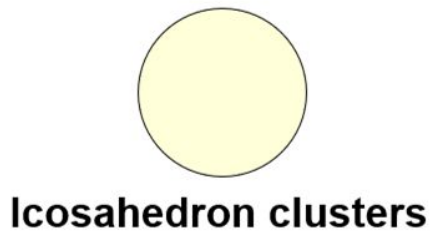

Icosahedron clusters

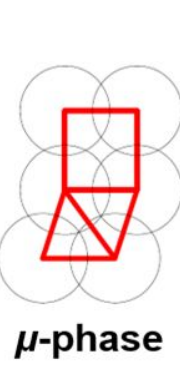

$C_{14}$-phase

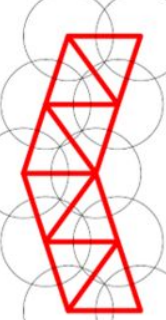

$C_{36}$-phase

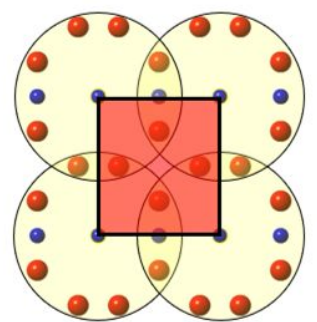

Z-phase, [010]
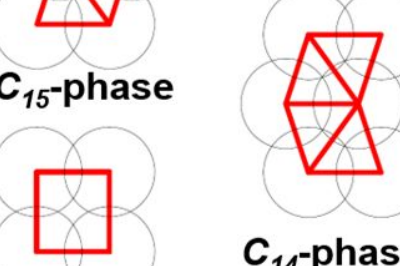

Z-phase

$C_{15}$-phase
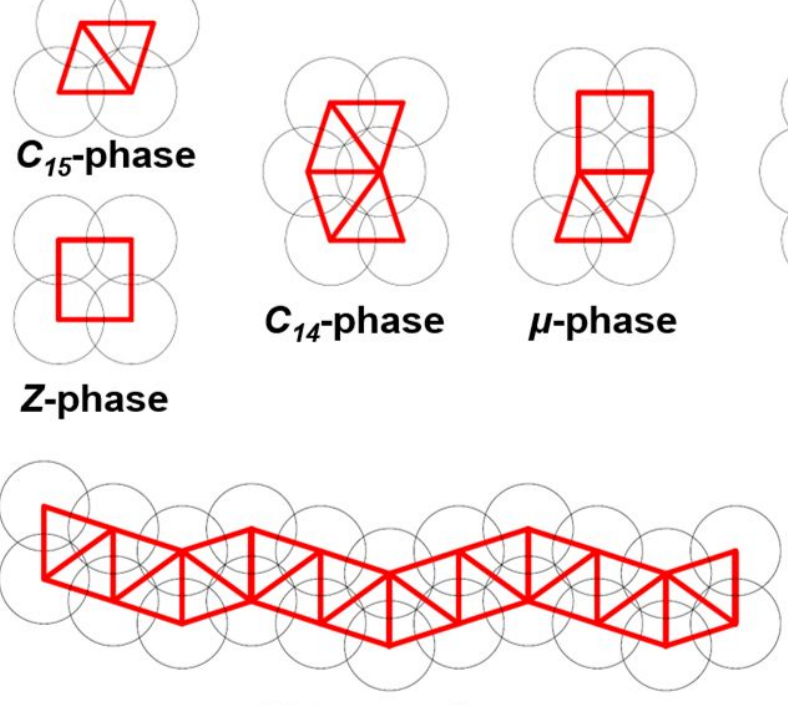

10-layers phase

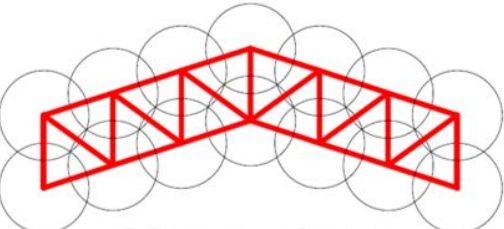

6-layers phase

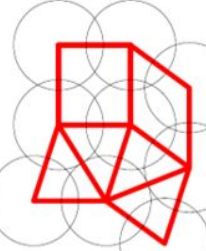

C-phase

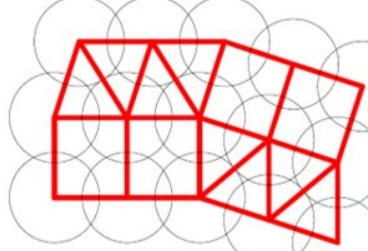

M-phase

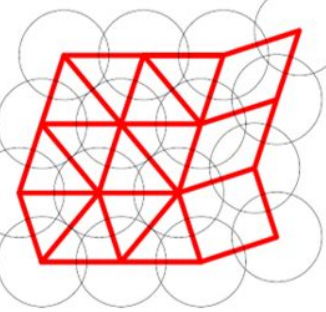

mz-phase

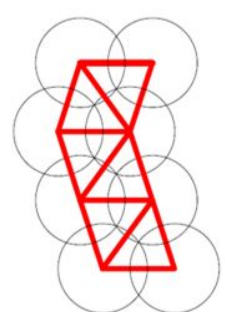

9-layers phase

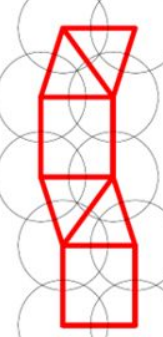

zra-d-phase

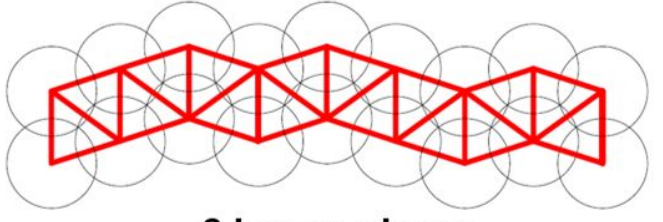

8-layers phase

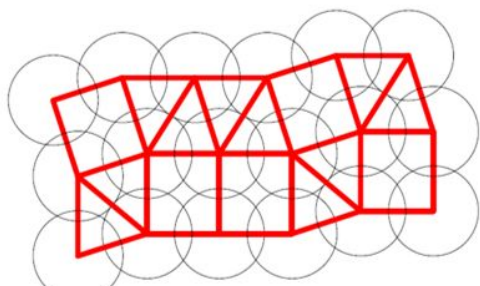

I-phase

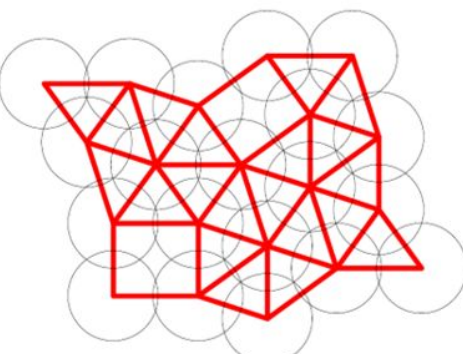

$X$-phase

Figure S1. Schematic diagrams of the pentagonal Frank-Kasper phases, which assembled by the $C_{15}$ and $Z$ plane tiling patterns. And it can be found that these Frank-Kasper phases are arranged along the pseudo ten-fold axis by the icosahedron columns. 


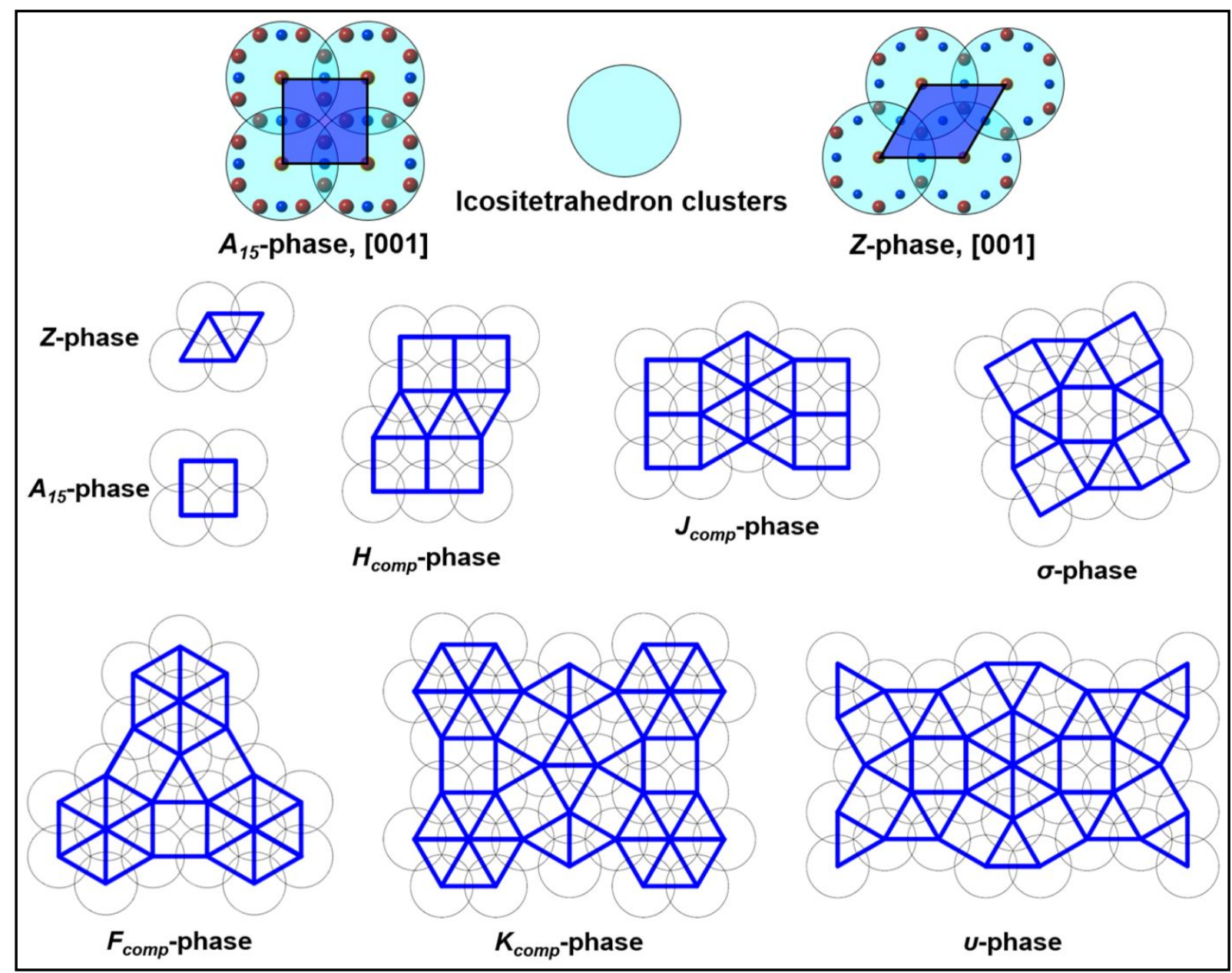

Figure S2. Schematic diagrams of the hexagonal Frank-Kasper phases, which assembled by the $A_{15}$ and $Z$ plane tiling patterns. And it can be found that these Frank-Kasper phases are arranged along the pseudo twelve-fold axis by the icositetrahedron columns. 

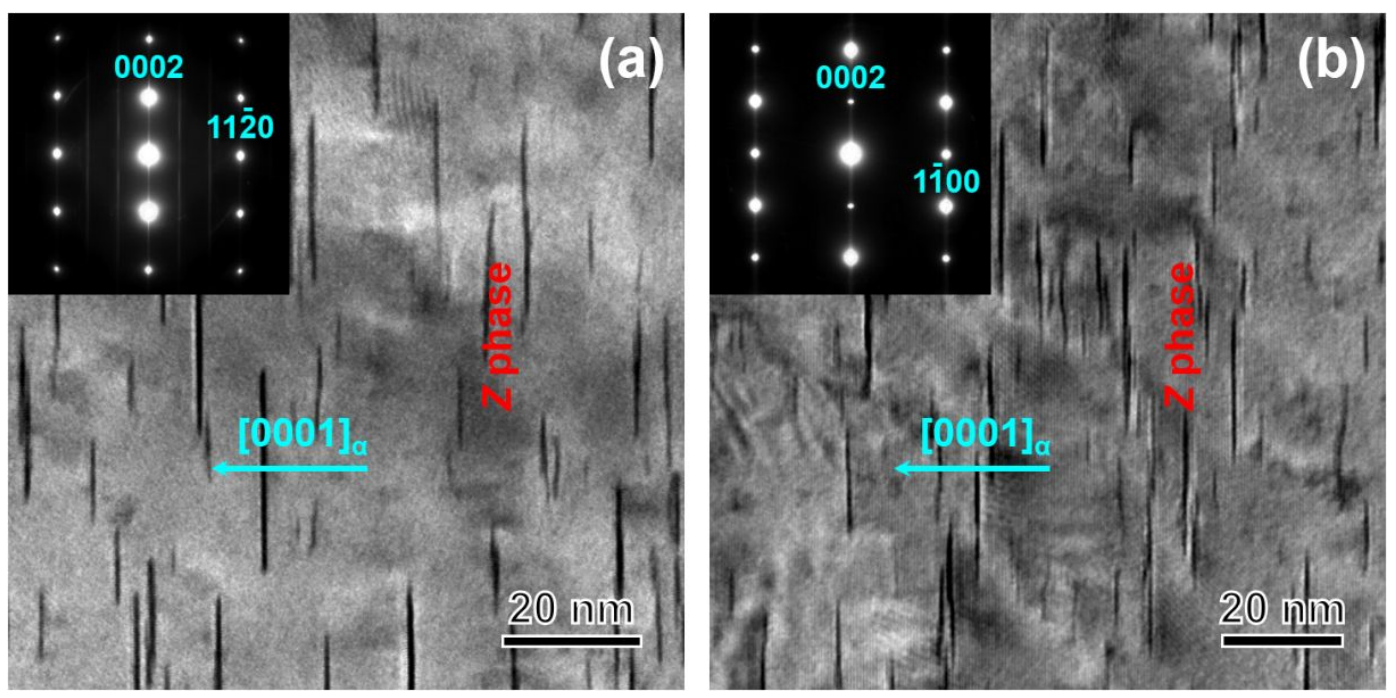

Figure S3. Bright-field images showing the 2D Frank-Kasper $Z$ phase precipitated in the Mg-4Sm-2Zn alloy after aged at $200{ }^{\circ} \mathrm{C}$ for $48 \mathrm{~h}$. The electron beam is parallel to the $[1 \overline{1} 00]_{\alpha}$ (a) and $[11 \overline{2} 0]_{\alpha}$ (b). The insets are the corresponding selected-area electron diffraction (SAED) patterns. 


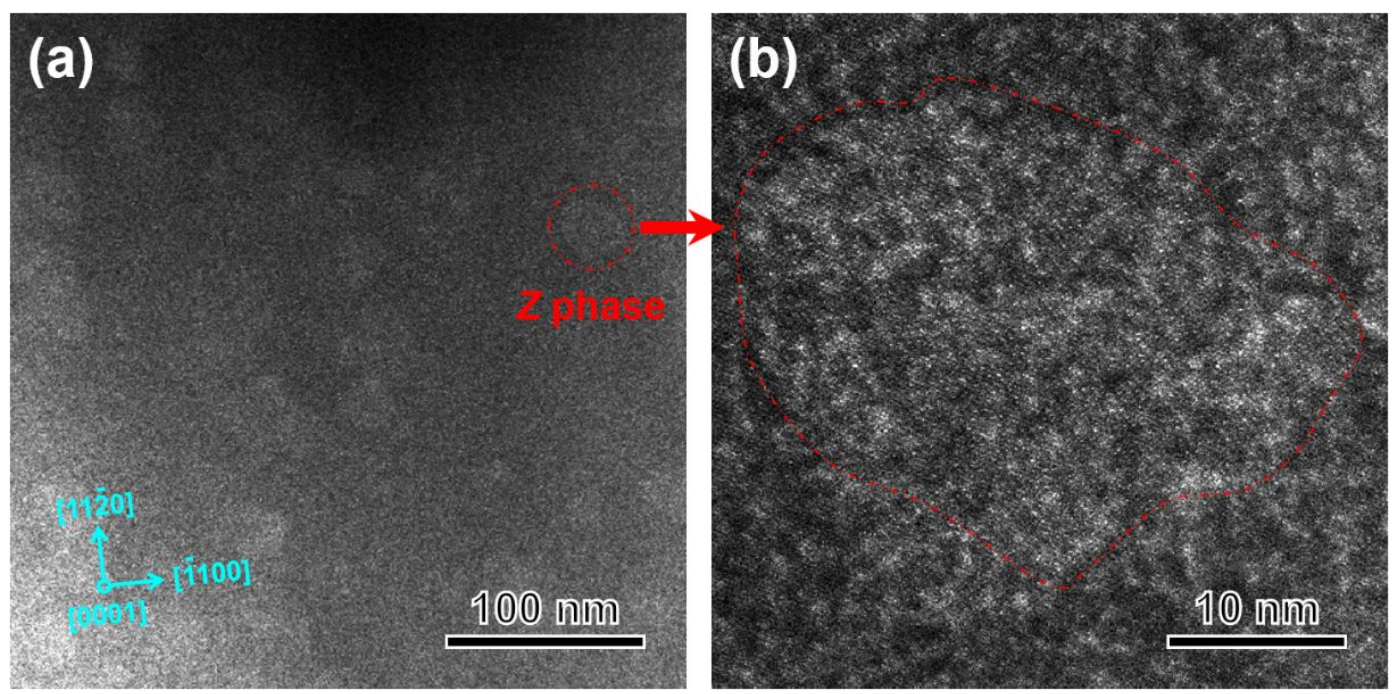

Figure S4. HAADF-STEM images of the 2D Frank-Kasper $Z$ phase in a Mg-4Sm-2Zn (wt.\%) alloy after aged at 200 ${ }^{\circ} \mathrm{C}$ for $48 \mathrm{~h}$. The electron beam is parallel to the $[0001]_{\alpha}$. 


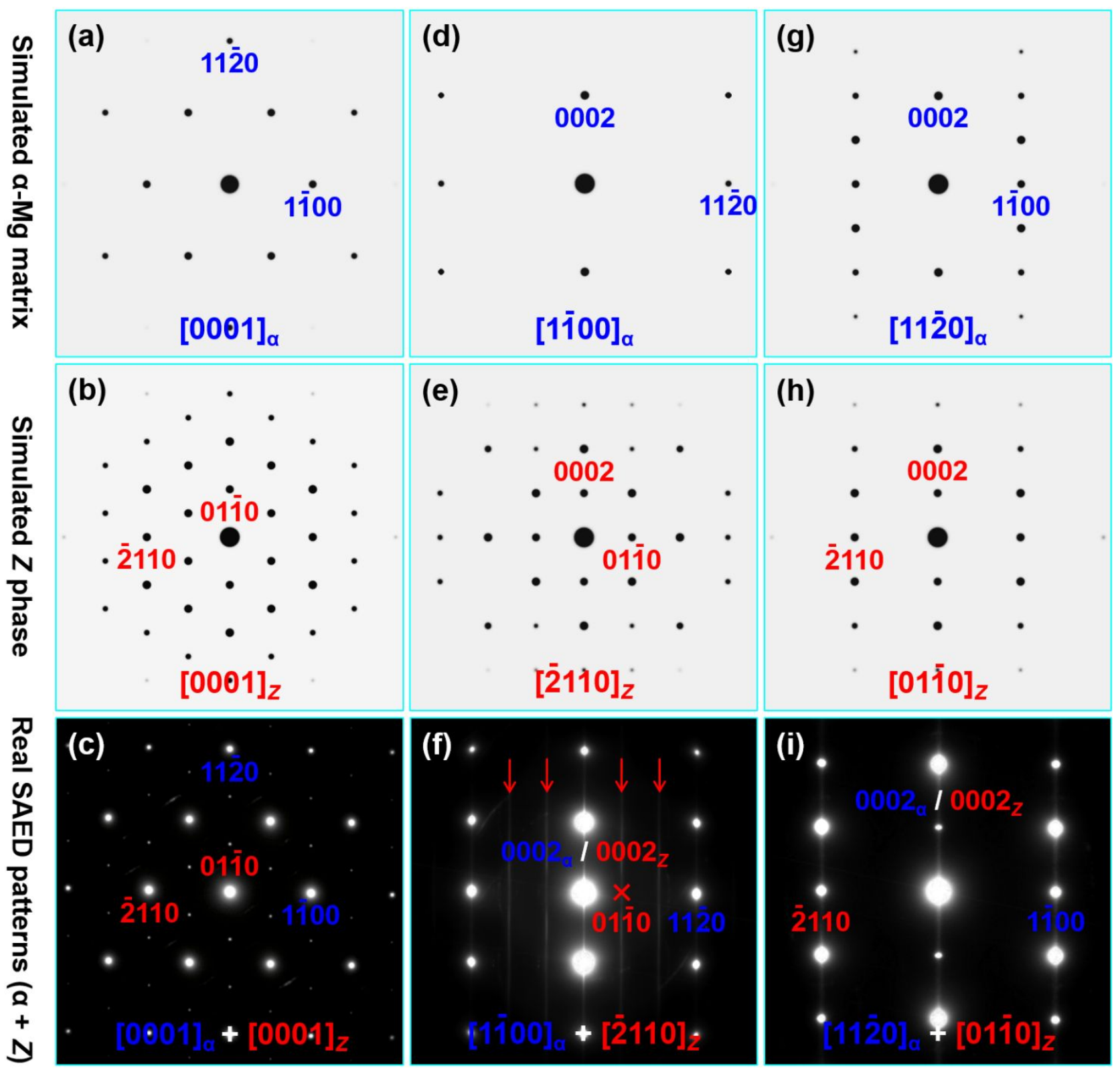

Figure S5. Electron diffraction results. (a, d, and g) Simulated electron diffraction patterns of the $\alpha$-Mg matrix from $[0001]_{\alpha}(\mathrm{a}),[1 \overline{1} 00]_{\alpha}(\mathrm{d})$, and $[11 \overline{2} 0]_{\alpha}(\mathrm{g})$. (b, e, and h) Simulated electron diffraction prtterns of the 2D FrankKasper $Z-\mathrm{Mg}_{2} \mathrm{Sm}_{2} \mathrm{Zn}_{3}$ phase from $[0001]_{z}$ (b), $[\overline{2} 110]_{z}$ (e), and $[01 \overline{1} 0]_{z}$ (h). (c, f, and i) Select-area electron diffraction (SAED) patterns of the 2D Frank-Kasper Z-Mg $\mathrm{Sm}_{2} \mathrm{Zn}_{3}$ precipitate in the $\mathrm{Mg}-\mathrm{Sm}-\mathrm{Zn}$ alloy from $[0001]_{\alpha+z}(\mathrm{c}),[1 \overline{1} 00]_{\alpha}+[\overline{2} 110]_{z}(\mathrm{f})$, and $[11 \overline{2} 0]_{\alpha}+[01 \overline{1} 0]_{z}(\mathrm{i})$. It can be found that the simulation results are consistent with the experimental results, in which without considering the $\{0001\}_{z}$ basal planes diffraction. Since $2 \mathrm{D}$ Frank-Kasper $Z-\mathrm{Mg}_{2} \mathrm{Sm}_{2} \mathrm{Zn}_{3}$ precipitate only has a single unit-cell height in the $[0001]_{z}$ direction, and it has also no periodicity along the $[0001]_{z}$ direction, the diffraction streaks parallel to the $[0001]_{z}$ direction are observed in the actual electron diffraction patterns. 


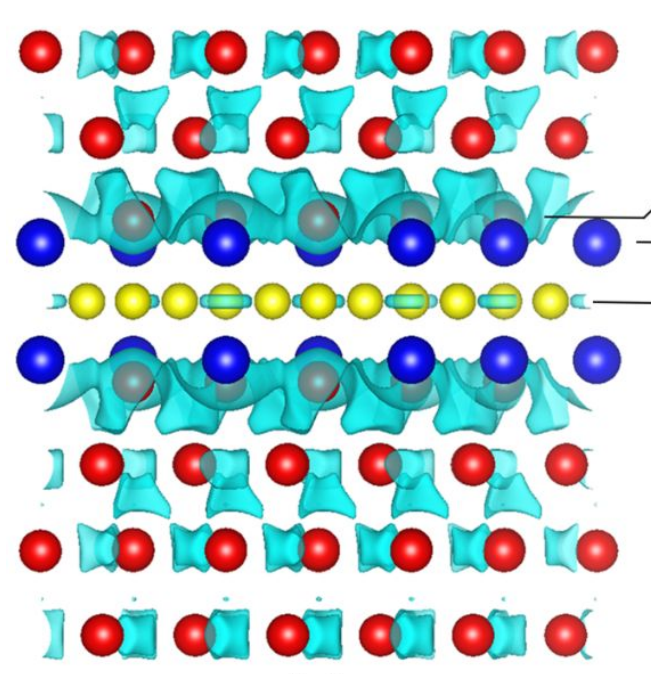

(a)

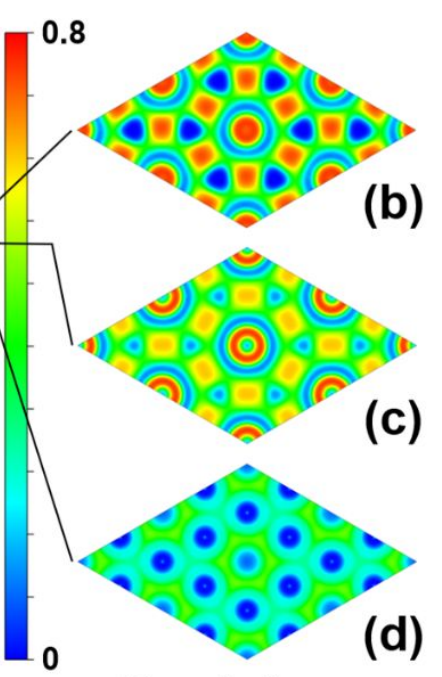

Basal planes

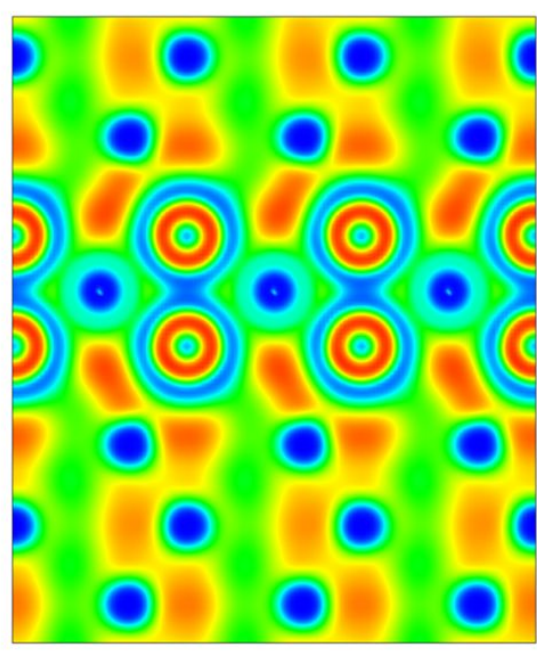

(e)

Figure S6. The first-principles computation results of the $2 \mathrm{D}$ Frank-Kasper $Z-\mathrm{Mg}_{2} \mathrm{Sm}_{2} \mathrm{Zn}_{3}$ phase. The direction is viewed along the $[1 \overline{1} 00]_{Z}$ (a and e) and $[0001]_{Z}(\mathrm{~b}-\mathrm{d})$. (a) Charge distribution of the "BAB-A'CA'-BA" sandwiched model. (b-e) The corresponding electron localization function distribution for the slices of $\{0001\}_{Z}(b-d)$ and $\{1 \overline{1}$ $00\}_{Z}(\mathrm{e})$ sections. The charge iso-surface setting using the $\Delta \rho=0.015 \mathrm{e} \cdot \AA^{-3}$ in the panels. 

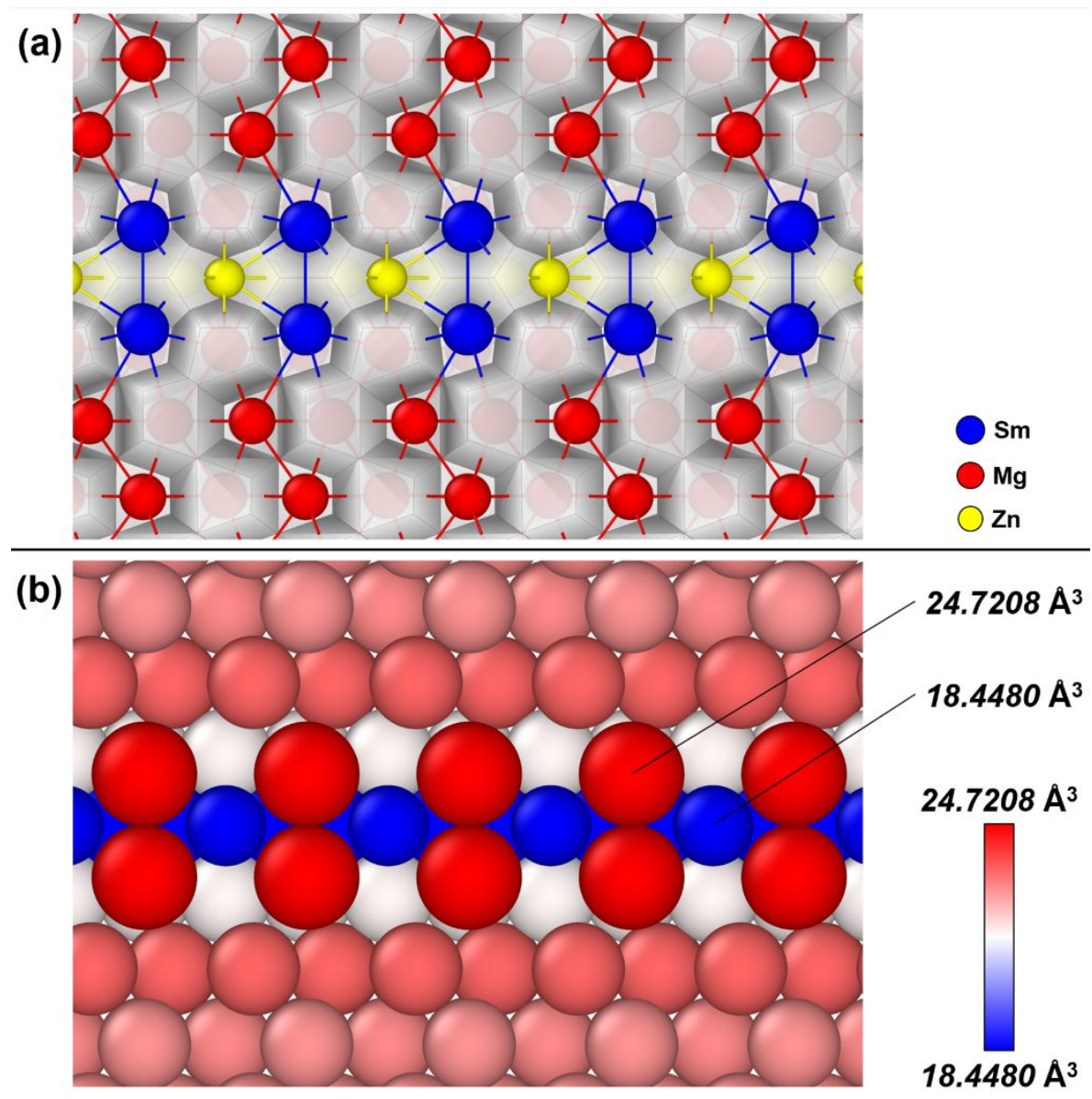

Figure S7. Voronoi analysis showing the 2D Frank-Kasper $Z-\mathrm{Mg}_{2} \mathrm{Sm}_{2} \mathrm{Zn}_{3}$ phase possesses a volume asymmetry ratio of 1.340. (a) Voronoi ployhedra analysis; (b) Voronoi analysis map. 

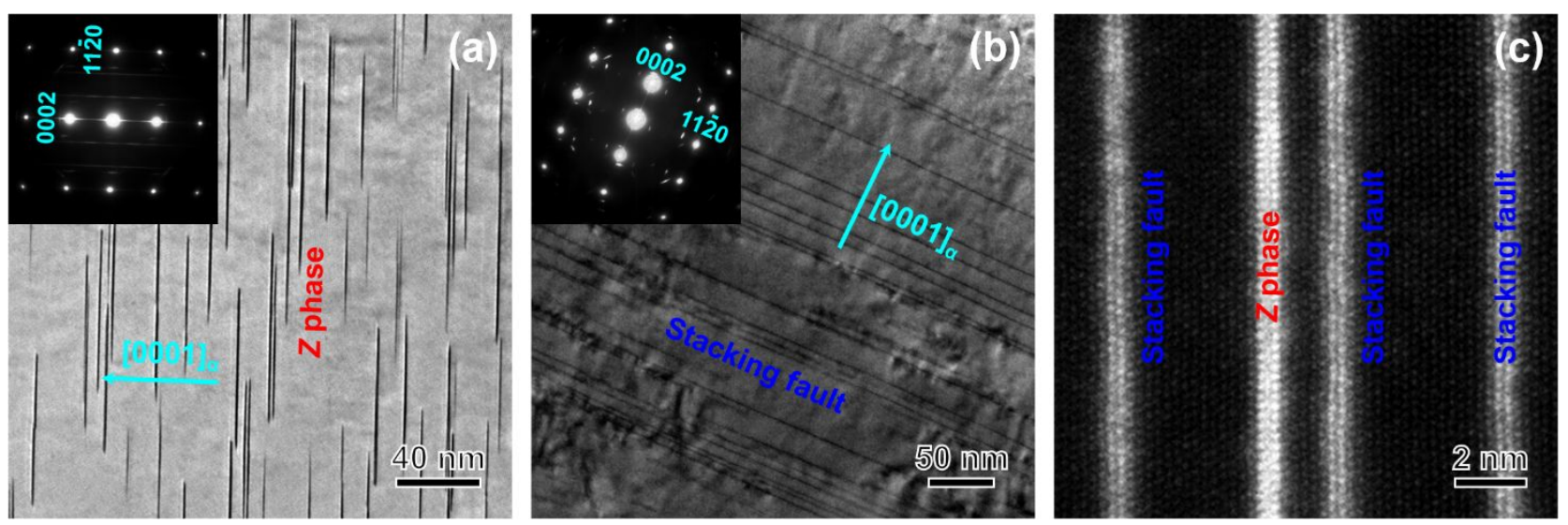

Figure S8. (a) Bright-field image of the Mg-4Sm-2Zn alloy after aged at $20{ }^{\circ} \mathrm{C}$ for $100 \mathrm{~h}$. (b) Bright-field image of the Mg-4Sm-2Zn alloy subjected to continuous isothermal aging at $250^{\circ} \mathrm{C}$ for $2 \mathrm{~h}$. (c) HAADF-STEM image of the Mg-4Sm-2Zn alloy subjected to continuous isothermal aging at $250{ }^{\circ} \mathrm{C}$ for $2 \mathrm{~h}$. The electron beam is parallel to the $[1 \overline{1} 00]_{\alpha}(\mathrm{a}$ and $\mathrm{b})$ and $[11 \overline{2} 0]_{\alpha}$ (c) directions. The insets are the corresponding SAED patterns. 


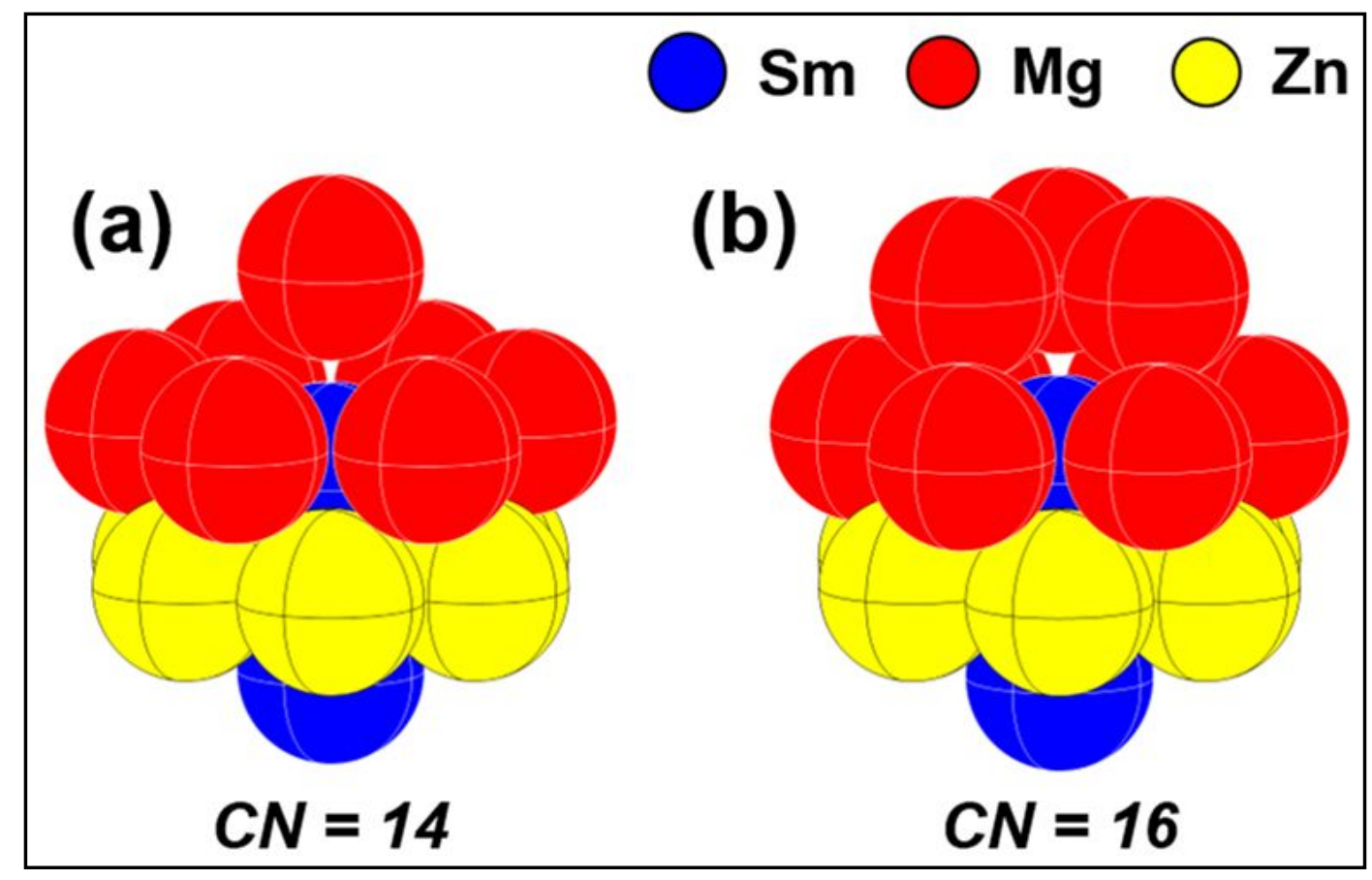

Figure S9. Atomic cluster models: (a) $C N=14$, icositetrahedron; (b) $C N=16$, Friauf polyhedron. 


\section{Supplementary Tables}

Table S1. Crystallographic parameters of the Frank-Kasper phases ${ }^{[1-24]}$

\begin{tabular}{|c|c|c|c|c|c|c|c|c|c|c|c|c|c|c|}
\hline \multirow{2}{*}{ No. } & \multirow{2}{*}{ Phase } & \multirow{2}{*}{$\begin{array}{c}\text { Representative } \\
\text { alloy }\end{array}$} & \multirow{2}{*}{ Space group } & \multicolumn{4}{|c|}{ Lattice parameters $(\AA)$} & \multirow{2}{*}{ Atomic Number } & \multicolumn{5}{|c|}{$C N(\%)$} & \multirow{2}{*}{ Ref. } \\
\hline & & & & $a$ & $b$ & $c$ & $\beta\left({ }^{\circ}\right)$ & & 16 & 15 & 14 & 12 & A. $V$. & \\
\hline 1 & $\overline{A_{15}}$ & $\mathrm{Cr}_{3} \mathrm{Si}$ & $P m^{\overline{3}} n$ & 4.560 & 4.560 & 4.560 & & 8 & 0 & 0 & 75.0 & 25.0 & 13.50 & [1] \\
\hline 2 & $\sigma$ & $\mathrm{Cr}_{46} \mathrm{Fe}_{54}$ & $\mathrm{P} 4_{2} / \mathrm{mnm}$ & 8.797 & 8.797 & 4.559 & & 30 & 0 & 13.3 & 53.4 & 33.3 & 13.47 & [2] \\
\hline 3 & $\mathrm{H}$ & Complex & $\mathrm{Cmmm}$ & 4.500 & 17.500 & 4.500 & & 30 & 0 & 13.3 & 53.4 & 33.3 & 13.47 & {$[3,4]$} \\
\hline 4 & $\mathrm{~K}$ & Complex & Pmmm & 12.500 & 17.100 & 4.500 & & 82 & 0 & 14.6 & 51.2 & 34.2 & 13.46 & {$[3,4]$} \\
\hline 5 & $\mathrm{~F}$ & Complex & P6/mmm & 12.500 & 12.500 & 4.500 & & 52 & 0 & 15.4 & 50.0 & 34.6 & 13.46 & {$[3,4]$} \\
\hline 6 & $\mathrm{~J}$ & Complex & Pmmm & 4.500 & 12.500 & 4.500 & & 22 & 0 & 18.2 & 45.5 & 36.3 & 13.46 & {$[3,4]$} \\
\hline 7 & $v$ & $\mathrm{Mn}_{81.5} \mathrm{Si}_{18.5}$ & Immm & 16.992 & 28.634 & 4.656 & & 186 & 6.5 & 10.5 & 43.0 & 40.0 & 13.43 & [5] \\
\hline 8 & $\mathbf{Z}$ & $\mathbf{Z r}_{4} \mathbf{A l}_{3}$ & $P 6 / m m m$ & 5.433 & 5.433 & 5.390 & & 7 & 0 & 28.6 & 28.6 & 42.8 & 13.43 & [6] \\
\hline 9 & $\mathrm{P}$ & $\mathrm{Mo}_{42} \mathrm{Cr}_{18} \mathrm{Ni}_{40}$ & Pbnm & 9.07 & 17.01 & 4.74 & & 56 & 7.1 & 14.3 & 35.7 & 42.9 & 13.43 & [7] \\
\hline 10 & $\delta$ & MoNi & $P 2{ }_{1} 2_{l} 2_{l}$ & 9.108 & 9.108 & 8.852 & & 56 & 7.1 & 14.3 & 35.7 & 42.9 & 13.43 & [8] \\
\hline 11 & $\mathrm{~K}$ & $\mathrm{Mn}_{77} \mathrm{Fe}_{4} \mathrm{Si}_{19}$ & $C 2$ & 13.362 & 11.645 & 8.734 & 90.5 & 220 & 12.7 & 7.3 & 34.5 & 45.5 & 13.41 & [9] \\
\hline 12 & $\mathrm{R}$ & $\mathrm{Mo}_{31} \mathrm{Cr}_{51} \mathrm{Co}_{18}$ & $R^{\overline{3}}$ & 10.903 & 10.903 & 19.342 & & 159 & 15.1 & 11.3 & 22.6 & 51.0 & 13.40 & {$[10]$} \\
\hline 13 & $\mu$ & $\mathrm{Mo}_{6} \mathrm{Co}_{7}$ & $R^{\overline{3}_{m}}$ & 4.762 & 4.762 & 25.615 & & 39 & 15.4 & 15.4 & 15.4 & 53.8 & 13.39 & {$[11]$} \\
\hline 14 & zra-d & $\mathrm{K}_{7} \mathrm{Cs}_{6}$ & $\mathrm{P}_{3} / \mathrm{mmc}$ & 9.078 & 9.078 & 32.950 & & 26 & 15.4 & 15.4 & 15.4 & 53.8 & 13.39 & [12] \\
\hline 15 & $\mathrm{P}_{\sigma}$ & $\mathrm{W}_{6}(\mathrm{Fe}, \mathrm{Si})_{7}$ & Pbam & 9.283 & 7.817 & 4.755 & & 26 & 15.4 & 15.4 & 15.4 & 53.8 & 13.39 & [13] \\
\hline 16 & M & $\mathrm{Nb}_{48} \mathrm{Ni}_{39} \mathrm{Al}_{13}$ & Pnma & 9.303 & 4.933 & 16.266 & & 52 & 15.4 & 15.4 & 15.4 & 53.8 & 13.39 & {$[14]$} \\
\hline 17 & I & $\mathrm{V}_{41} \mathrm{Ni}_{36} \mathrm{Si}_{23}$ & $\mathrm{Cc}$ & 13.462 & 23.381 & 8.940 & 100.3 & 228 & 21.1 & 10.5 & 10.5 & 57.9 & 13.37 & {$[15]$} \\
\hline 18 & $\mathrm{C}$ & $\mathrm{V}_{2}(\mathrm{Co}, \mathrm{Si})_{3}$ & $C 2 / m$ & 17.170 & 4.660 & 17.62 & 155 & 50 & 24.0 & 8.0 & 8.0 & 60.0 & 13.36 & [16] \\
\hline 19 & $\mathrm{~T}$ & $\mathrm{Mg}_{32}(\mathrm{Zn}, \mathrm{Al})_{49}$ & $I m^{\overline{3}}$ & 14.160 & 14.160 & 14.160 & & 162 & 24.7 & 7.4 & 7.4 & 60.5 & 13.36 & {$[17]$} \\
\hline 20 & $\mathrm{X}$ & $\mathrm{Mn}_{45} \mathrm{Co}_{40} \mathrm{Si}_{15}$ & Pnnm & 15.500 & 12.470 & 4.760 & & 74 & 27.0 & 5.4 & 5.4 & 62.2 & 13.35 & {$[18]$} \\
\hline 21 & $\mathrm{mz}$ & $\mathrm{Mg}_{4} \mathrm{Zn}_{7}$ & $C 2 / m$ & 25.960 & 5.240 & 14.280 & 102.5 & 110 & 29.1 & 3.6 & 3.6 & 63.7 & 13.34 & [19] \\
\hline 22 & 6-layers & $\mathrm{MgCuNi}$ & $\mathrm{P}_{3} / \mathrm{mmc}$ & 4.917 & 4.917 & 24.040 & & 36 & 33.3 & 0 & 0 & 66.7 & 13.33 & {$[20]$} \\
\hline 23 & 8-layers & $\mathrm{MgZn}_{2}+0.03 \mathrm{MgAg}_{2}$ & $\mathrm{PO}_{3} / \mathrm{mmc}$ & 5.210 & 5.210 & 34.400 & & 48 & 33.3 & 0 & 0 & 66.7 & 13.33 & {$[21]$} \\
\hline 24 & 9-layers & $\mathrm{MgZn}_{2}+0.07 \mathrm{MgAg}_{2}$ & $R^{\overline{3}_{m}}$ & 5.210 & 5.210 & 38.700 & & 54 & 33.3 & 0 & 0 & 66.7 & 13.33 & {$[21]$} \\
\hline 25 & 10-layers & $\mathrm{MgZn}_{2}+0.1 \mathrm{MgAg}_{2}$ & $\mathrm{~Pb}_{3} / \mathrm{mmc}$ & 5.220 & 5.220 & 43.000 & & 60 & 33.3 & 0 & 0 & 66.7 & 13.33 & {$[21]$} \\
\hline 26 & $\mathrm{C}_{14}$ & $\mathrm{MgZn}_{2}$ & $\mathrm{P}_{3} / \mathrm{mmc}$ & 5.223 & 5.223 & 8.566 & & 12 & 33.3 & 0 & 0 & 66.7 & 13.33 & {$[22]$} \\
\hline 27 & $\mathrm{C}_{36}$ & $\mathrm{MgNi}_{2}$ & $\mathrm{P}_{3} / \mathrm{mmc}$ & 4.824 & 4.824 & 15.826 & & 24 & 33.3 & 0 & 0 & 66.7 & 13.33 & [23] \\
\hline 28 & $\mathrm{C}_{15}$ & $\mathrm{MgCu}_{2}$ & $F d^{\overline{3}} m$ & 7.061 & 7.061 & 7.061 & & 24 & 33.3 & $\mathbf{0}$ & $\mathbf{0}$ & 66.7 & 13.33 & [24] \\
\hline
\end{tabular}

\section{References}

(1) Jauch, W.; Schultz, A. J.; Heger, G. Single-crystal time-of-flight neutron diffraction of $\mathrm{Cr}_{3} \mathrm{Si}$ and $\mathrm{MnF}_{2}$ : comparison with monochromatic-beam techniques. J Appl. Crystallog. 1987, 20, 117-119.

(2) Yakel, H. L. jr. Atom distribution in sigma-phases. I. Fe and $\mathrm{Cr}$ distribution in a binary phase equilibrated at 1063, 1013, and 923 K. Acta Crystallog. B 1983, 39, 20-28.

(3) Ye, H. Q.; Li, D. X.; Guo, K. X. Topologically closed-packed phase in superalloys: New phase and domain structures. Acta Metal. Sin. 1986, 22, A1-A43.

(4) Dutour Sikiric, M.; Delgado-Friedrichs, O.; Deza, M. Space fullerenes: a computer search for new Frank-Kasper structures. Acta Crystallog. A 2010, A66, 602-615.

(5) Brink-Shoemaker, C.; Shoemaker, D. P. The Crystal Structure of the mue Phase, $\mathrm{Mn}_{81.5} \mathrm{Si}_{18.5}$. Acta Crystallog. B 1971, 27, 227-235.

(6) Wilson, C. G.; Thomas, D. K.; Spooner, F. J. The crystal structure of $\mathrm{Zr}_{4} \mathrm{Al}_{3}$. Acta Crystallog. 1960, 13, 56-57.

(7) Shoemaker, C. B.; Shoemaker, D. P. A variation on the sigma-phase structure: the structure of the P phase, MoNi-Cr. Acta Crystallog. 1955, 8, 734-735.

(8) Shoemaker, C. B.; Shoemaker, D. P. The crystal structure of the delta phase, Mo-Ni. Acta Crystallog. 1963, 16, 997-1009.

(9) Shoemaker, B.; Shoemaker, D. Crystal structure and superstructure of the k phase, $\mathrm{Mn}_{77} \mathrm{Fe}_{4} \mathrm{Si}_{19}$. Acta Crystallog. 
B 1977, 33, 743-754.

(10) Komura, Y.; Sly, W. G.; Shoemaker, D. P. The crystal structure of the R-phase, Mo-Co-Cr. Acta Crystallog. 1960, 13, 575-585.

(11) Forsyth, J. B.; D'Alte da Veiga, L. M. The structure of the mue-phase $\mathrm{Co}_{7} \mathrm{Mo}_{6}$. Acta Crystallog. 1962, 15, 543546.

(12) Simon, von A.; BraMer, W.; HillenkoTter, B.; Kullmann, H. J. Neue Verbindungen zwischen Kalium und Caesium. Zeitschrift fuer Anorganische und Allgemeine Chemie 1976, 419, 253-274.

(13) Kripyakevich, P. I.; Yarmolyuk, Ya. P. The crystal structure of $\mathrm{W}_{2} \mathrm{FeSi}=\mathrm{W}_{6}\left(\mathrm{~W}_{0.07} \mathrm{Fe}_{0.465} \mathrm{Si}_{0.465}\right)_{7}$. Dopovidi Akademii Nauk Ukrains'koi RSR, Seriya A: Fiziko-Matematichni Ta Tekhnichni Nauki 1974, 36, 460-463.

(14) Shoemaker, C. B.; Shoemaker, D. P. The crystal structure of the M phase, Nb-Ni-Al. Acta Crystallog. 1967, 23, 231-238.

(15) Brink-Shoemaker, C.; Shoemaker, D. P. The structure of the I phase, $\mathrm{V}_{41} \mathrm{Ni}_{36} \mathrm{Si}_{23}$, a pseudo superstructure. Acta Crystallog. B 1981, 37, 1-8.

(16) Kripyakevich, P. I.; Yarmolyuk, Ya. P. Crystal structure of the C-phase $\mathrm{V}_{2}\left(\mathrm{Co}_{0.57} \mathrm{Si}_{0.43}\right)_{3}$ - new example of the structure of $\mathrm{Zr}_{4} \mathrm{Al}_{3}-\mathrm{MgZn}_{2}$ homologous series. Dopovidi Akademii Nauk Ukrains'koi RSR, Seriya A: FizikoTekhnichni ta Matematichni Nauki 1970, 32, 948-950.

(17) Bergman, G.; Waugh, J. L. T.; Pauling, L. The crystal structure of the metallic phase $\mathrm{Mg}_{32}(\mathrm{Al}, \mathrm{Zn})_{49} \mathrm{Al}-\mathrm{Zn}-\mathrm{Mg}$ alloys. Acta Crystallog. 1957, 10, 254-259.

(18) Yarmolyuk, Ya. P.; Kripyakevich, P. I.; Gladyshevskii, E. I. The crystal structure of the X-phase in the Mn-CoSi system. Kristallografiya 1970, 15, 268-274.

(19) Yarmolyuk, Ya. P.; Kripyakevich, P. I.; Mel'nik, E. V. Crystal structure of the compound $\operatorname{Mg}_{4} Z_{7}$. Kristallografiya 1975, 20, 538-542.

(20) Komura, Y.; Nakaue, A.; Mitarai, M. Crystal structure of a new stacking variant of a Friauf-Laves phase in the system Mg-Cu-Ni. Acta Crystallog. B 1972, 28, 727-732.

(21) Komura, Y.; Tokunaga, K. Structural studies of stacking variants in Mg-base Friauf-Laves-phases. Acta Crystallog. B 1980, 36, 1548-1554.

(22) Ohba, T.; Kitano, Y.; Komura, Y. The charge-density study of the Laves phases, $\mathrm{MgZn}_{2}$ and $\mathrm{MgCu}_{2}$. Acta Crystallog. C 1984, 40, 1-5.

(23) Komura, Y.; Tokunaga, K. Structural studies of stacking variants in Mg-base Friauf- Laves phases. Acta Crystallog. B 1980, 36, 1548-1554.

(24) Braga, M. H.; Ferreira, J. J. A.; Siewenie, J.; Proffen, T.; Daemen, L. L. Neutron powder diffraction and firstprinciples computational studies of $\mathrm{CuLi}(\mathrm{x}) \mathrm{Mg}(2-\mathrm{x})(\mathrm{x} \sim=0.08), \mathrm{CuMg}_{2}$, and $\mathrm{Cu}_{2} \mathrm{Mg}$. J Solid State Chem. 2010, $183,10-19$. 
Table S2. Structure parameters of the 2D Frank-Kasper $Z-\mathrm{Mg}_{2} \mathrm{Sm}_{2} \mathrm{Zn}_{3}$ phase.

\begin{tabular}{|c|c|c|c|c|c|c|c|}
\hline $\begin{array}{l}\text { Chemical } \\
\text { formula }\end{array}$ & Atom number in cell & Space group & Lattice parameter & Atom site & $\begin{array}{l}\text { Largest Voronoi } \\
\text { volume }\end{array}$ & $\begin{array}{l}\text { Largest Voronoi } \\
\text { volume }\end{array}$ & $\begin{array}{c}\text { Volume } \\
\text { ratio }\end{array}$ \\
\hline $\mathrm{Mg}_{2} \mathrm{Sm}_{2} \mathrm{Zn}_{3}$ & 7 & P6/mmm & $\begin{array}{c}\alpha=\beta=90^{\circ}, \gamma=120^{\circ} \\
a=5.66 \AA, c=4.85 \AA\end{array}$ & $\begin{array}{c}\text { Mg: 2c }(0.3333,0.6667,0) \\
\text { Sm: } 2 \mathrm{e}(0,0,0.1238) \\
\text { Zn: } 6 \mathrm{i}(0.500,0,0.500)\end{array}$ & $24.7208 \AA^{3}$ & $18.4480 \AA^{3}$ & 1.3400 \\
\hline
\end{tabular}

Table S3. First-principles computation results for the 2D Frank-Kasper $Z-\mathrm{Mg}_{2} \mathrm{Sm}_{2} \mathrm{Zn}_{3}$ phase.

\begin{tabular}{cccccc}
\hline \multirow{2}{*}{ No. } & \multirow{2}{*}{ Stacking model } & Formation & \multicolumn{3}{c}{ Lattice parameter $(\boldsymbol{A})$} \\
& & energy (meV/atom) & $\boldsymbol{a}$ & $\boldsymbol{c}$ & $\boldsymbol{H}_{\text {Sm-Sm }}$ \\
\hline $0+0$ & -A'CA'- & 38.54 & 5.50 & 5.18 & 3.31 \\
$1+2$ & B-A'CA'-BA & -117.85 & 5.58 & 4.85 & 3.52 \\
$\mathbf{2 + 3}$ & AB-A'CA'-BAB & $\mathbf{- 8 8 . 1 9}$ & $\mathbf{5 . 5 7}$ & $\mathbf{4 . 8 5}$ & $\mathbf{3 . 5 3}$ \\
$2+3$ & BA-A'CA'-ABA & -18.79 & 5.50 & 5.01 & 3.33 \\
$3+4$ & BAB-A'CA'-BABA & -69.52 & 5.56 & 4.86 & 3.53 \\
$4+5$ & ABAB-A'CA'-BABAB & -58.09 & 5.55 & 4.88 & 3.52 \\
$5+6$ & BABAB-A'CA'-BABABA & -50.52 & 5.55 & 4.87 & 3.53 \\
$6+7$ & ABABAB-A'CA'-BABABAB & -44.26 & 5.56 & 4.87 & 3.50 \\
\hline
\end{tabular}

Table S4. Distances between the central atoms and adjacent atoms in the clusters.

3D views of the atomic clusters
Mg: red spheres; $S m$ : blue spheres; Zn: yellow spheres $\quad \begin{gathered}\text { Interatomic distances }(\AA) \\ \text { Atomic radii: } 1.60 \AA \text { for } \mathrm{Mg}, 1.80 \AA \text { for } \mathrm{Sm}, 1.39 \AA \text { for Zn }\end{gathered}$

